\title{
HALLAZGO IMAGENOLÓGICO DE HENDIDURA MANDIBULAR MEDIA INCOMPLETA: REPORTE DE UN CASO
}

\section{IMAGING FINDINGS OF AN INCOMPLETE MEDIAN MANDIBULAR CLEFT: A CASE REPORT}

\author{
Adalsa Hernández-Andara* \\ Ana I. Ortega-Pertuz** \\ Carina González***
}

\begin{abstract}
RESUMEN
La hendidura mandibular media es una rara anomalía congénita la cual ha sido descrita como hendidura Tessier 30, la misma puede estar asociada a una variedad de alteraciones de distinta severidad y por ello expresarse como una simple muesca en el bermellón del labio hasta la ausencia del manubrio esternal. El presente trabajo tuvo como propósito describir un hallazgo imagenológico de hendidura mandibular media incompleta, con énfasis en el recurso de la Tomografía Computarizada (TC) como medio para su caracterización. Paciente masculino de 11 años de edad quien es referido para estudio mediante TC, por la presencia de una imagen radiolúcida en radiografía panorámica previa. Al examen extrabucal, no presentaba herida cutánea o asimetría facial; en el examen intrabucal se observó la ausencia de elementos dentarios permanente en zona anteriosuperior e inferior. En la radiografía panorámica se verificó la retención de los dientes 12-22, 32, 42 y 41, así como una imagen radiolúcida en línea media de la mandíbula, de bordes corticalizados que se extendía desde el borde inferior sin alcanzar la cresta del reborde alveolar. Las reconstrucciones en los planos axial, sagital y coronal obtenidas por TC mostraron la extensión de la imagen hipodensa en sentido vestíbulo-lingual, la cual no ocupaba el espesor del hueso, comprobándose también la corticalización de los márgenes. El uso de la TC permitió conocer los límites y extensión de la alteración, así como confirmar la corticalización de los márgenes en la imagen, lo que permitió formular la hipótesis diagnóstica de hendidura mandibular media.
\end{abstract}

PALABRAS CLAVE: Mandíbula • Tomografía Computarizada por Rayos $X$

\section{ABSTRACT}

Median mandibular cleft is a rare congenital anomaly described as Tessier cleft 30, associated with a variety of disorders of different severity; it could be expressed as a notch in the lip vermillion to the absence of the sternal manubrium. The present study was aimed to describe an imaging finding of a median incomplete mandibular cleft, with emphasis on the use of computed tomography (CT) as a means of characterization. An 11 year old male patient referred for CT study, due to the presence of a radiolucent image in a previous panoramic radiograph. The extraoral examination showed no skin wound or facial asymmetry; intraoral exam demonstrated the absence of anterior superior and lower permanent tooth elements. In the panoramic radiograph the impaction of teeth $12-22,32,42$ and 41 , as well as a midline radiolucent image in the mandible with corticalized margins extending from the inferior border without reaching the alveolar ridge crest was verified. Reconstructions in the axial, sagittal and coronal planes obtained by CT showed extension of the hypodense image in the vestibular-lingual orientation, which did not occupy the entire bone thickness, also it was ascertained margins corticalization The use of CT allowed to know the limits and extent of the alteration, and confirm margins corticalization in the image, allowing to formulate a diagnostic hypothesis of incomplete median mandibular cleft.

KEY WORDS: Mandible • Tomography, X-Ray Computed

\footnotetext{
* Coordinadora de la Unidad de Imagen Dentomaxilofacial, Clínica Félix Boada, Caracas, Venezuela; Doctora en Diagnóstico Bucal, Sub-Área Radiología, FO/USP, adalsa1@yahoo.com.

** Profesora Titular, Área de Odontología Forense, Instituto de Investigaciones, Facultad de Odontología, Universidad del Zulia. Maracaibo, Estado Zulia Venezuela; Doctora en Odontología FACO/LUZ, Magíster en Radiología Odontológica FOP/UNICAMP, anitaortegav@gmail.com

*** Odontólogo; Facultad de Odontología, Universidad Santa María.
} 


\section{INTRODUCCIÓN}

Las hendiduras orofaciales constituyen las malformaciones faciales más comunes en todas las poblaciones y grupos étnicos. La hendidura facial tessier 30 es una rara alteración congénita que puede presentarse con un grado variable de severidad que va desde una muesca en el bermellón del labio inferior hasta la hendidura del manubrio esternal, en cuyo caso se involucraría la mandíbula, lengua, piso de la cavidad bucal, hueso hioides, cartílago tiroides y los haces musculares del cuello. No se han identificado factores hereditarios o predilección por sexo ${ }^{1-4}$.

La Tomografía Computadorizada (TC) ha permitido la valoración del complejo maxilofacial en los tres planos espaciales, lo que es de utilidad en el estudio de las hendiduras faciales, debido a que dichas alteraciones pueden caracterizarse en su extensión, localización, morfología y efectos en los órganos y tejidos adyacentes ${ }^{5,6}$. Considerando lo anteriormente expuesto, el presente trabajo tuvo como propósito reportar los hallazgos imagenológicos sugestivos de hendidura mandibular media encontrados en un paciente joven, enfatizando el uso de la TC para la evaluación tridimensional de la alteración.

\section{PRESENTACIÓN DEL CASO}

Paciente masculino de 11 años de edad quien acude a la Unidad de Imagen Dentomaxilofacial de la Clínica Félix Boada, Caracas, Venezuela, remitido para evaluación tomográfica por ausencia de unidades dentarias en la zona anterior de ambos maxilares, así como imagen radiolúcida en la sínfisis observada en radiografía panorámica previa, refiere caída reciente durante actividad deportiva.

Al examen extrabucal realizado en la Unidad de Imagen, el paciente no presentó alteraciones cutáneas o asimetría facial, mientras que al examen intrabucal se evidenció ausencia de los elementos dentarios permanentes $12-22,32,41$ y 42 . Los rebordes alveolares de la zona anterosuperior e inferior se observaron continuos, uniformes, con preservación de su altura, sin evidencia de expansión, la encía que los recubría mostraba un aspecto normal (Figura 1).

En la radiografía panorámica (Figura 2) se observó la presencia de los elementos dentarios temporarios 55-53, 65, 75-73, 84 y 85 y se evidenció rizálisis de 54, 53, 74, 73 y 84. Asimismo, se observaron los siguientes dientes permanentes en formación: 17, 15-13, 23,24, 27, 37, 35-33, 43-45, 47. Los dientes 12,11, 21 y 22 se encontraban retenidos. Las unidades dentarias $16,26,36$ y 46 se observaron erupcionadas, siendo que el 36 y 46 mostraron dilaceración y se encontraban en infra-oclusión. Asimismo, se evidenció la imagen de la cripta ósea del 38. Se evidenció una imagen radiolúcida de bordes definidos finamente corticalizados asociada a la corona del 46, similarmente, se evidenció imagen radiolúcida definida en relación con la corona del elemento dentario 37, ambas sugestivas de quiste dentígero. Se constató la presencia de imagen

Figura 1. a. Vista extrabucal del paciente y b. Vista inferior donde no se observan alteraciones cutáneas o asimetría; c. Vista frontal intrabucal donde se evidencia ausencia de elementos dentarios permanentes, en ambos maxilares, los rebordes alveolares se presentan continuos y uniformes, la encía que los recubre muestra una apariencia normal.

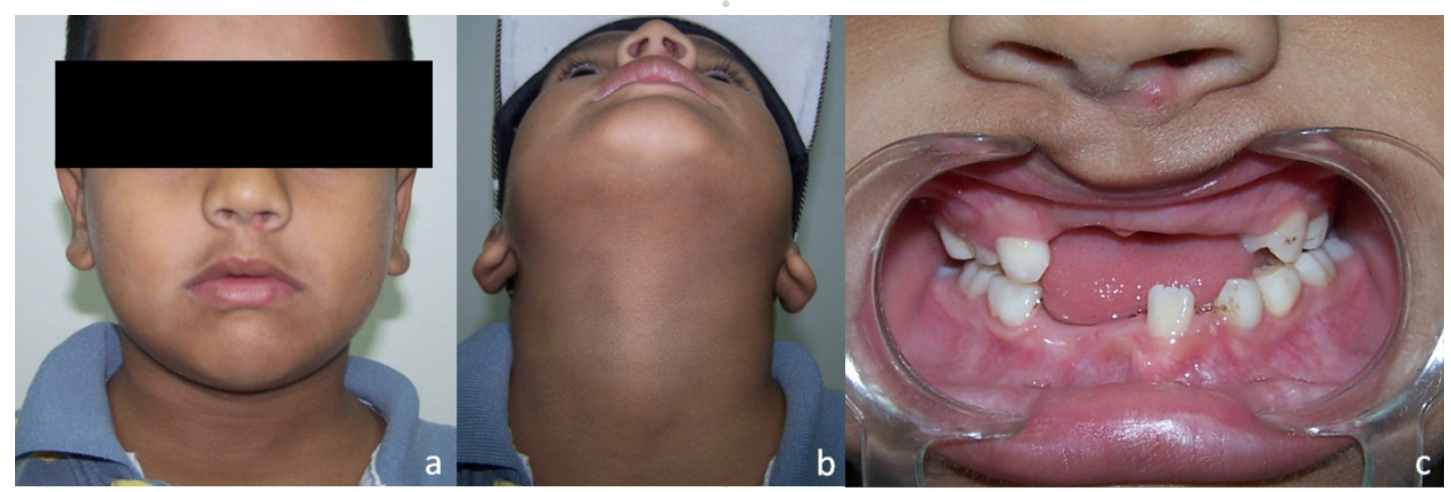

HERNÁNDEZ-ANDARA A ORTEGA-PERTUZ AI GONZÁLEZ C

HALLAZGO

IMAGENOLÓG I CO DE HENDIDURA

MANDIBULAR MEDIA INCOMPLETA :

REPORTE DE UN CASO
REV, ODONTOL.

UNIV. CID, SÃo

PAULO

2015; $27(2)$ :

156-62, MAIO-AGO 
HERNÁNDEZ-ANDARA A ORTEGA-PERTUZ AI GONZÁLEZ C

HALLAZGO IMAGENOLÓGICO DE HENDIDURA MANDIBULAR MEDIA INCOMPLETA: REPORTE DE UN CASO
Figura 2. Radiografía panorámica donde se obsęrva una imagen radiolúcida en región de sínfisis mentoniana, la cual se extiende desde el borde inferior de la mandíbula sin alcanzar la cresta alveolar. Nótese la retención de las unidades dentarias 32, 41 y 42, quienes están próximas a dicha imagen.

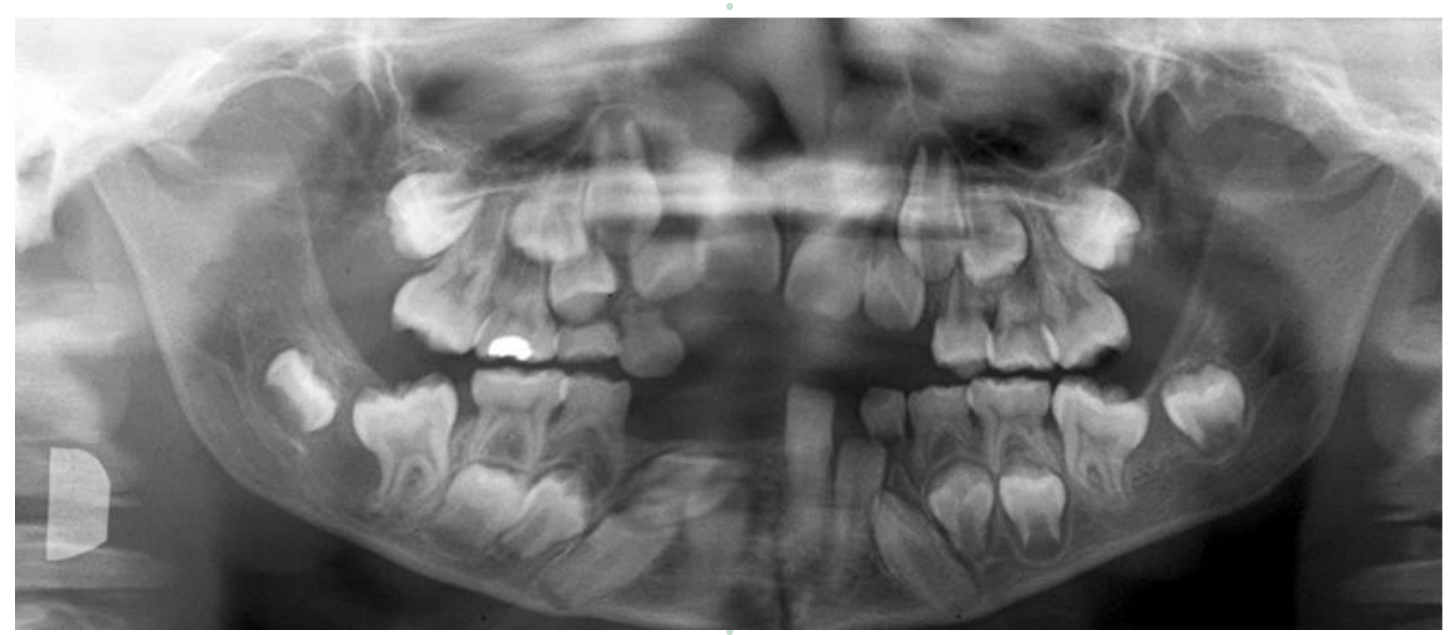

radiolúcida en sínfisis, que se extendía desde el borde inferior de la mandíbula sin alcanzar la cresta alveolar, de márgenes corticalizados.

Se realizó el examen de TC y se adquirieron imágenes volumétricas, reconstruidas en planos sagital, axial y coronal. En el plano axial (Figura 3) se evidenció una imagen hipodensa que se extendía desde la tabla ósea vestibular hacia la tabla ósea lingual, con márgenes corticalizados, siendo que en el corte más superior ilustrado en la Figura 3d esta imagen solo se evidencia como una solución de continuidad en la tabla ósea lingual.
En las reconstrucciones en el plano co-

Figura 3. Cortes axiales de tomografía computarizada donde 3a representa el corte más superior y el 3d el más inferior. Se evidencie de 3a a 3c, imagen hipodensa que se extiende desde la tabla ósea vestibular a la lingual, con márgenes corticalizados. En el corte 3d se observa solución de continuidad de la tabla ósea lingual.

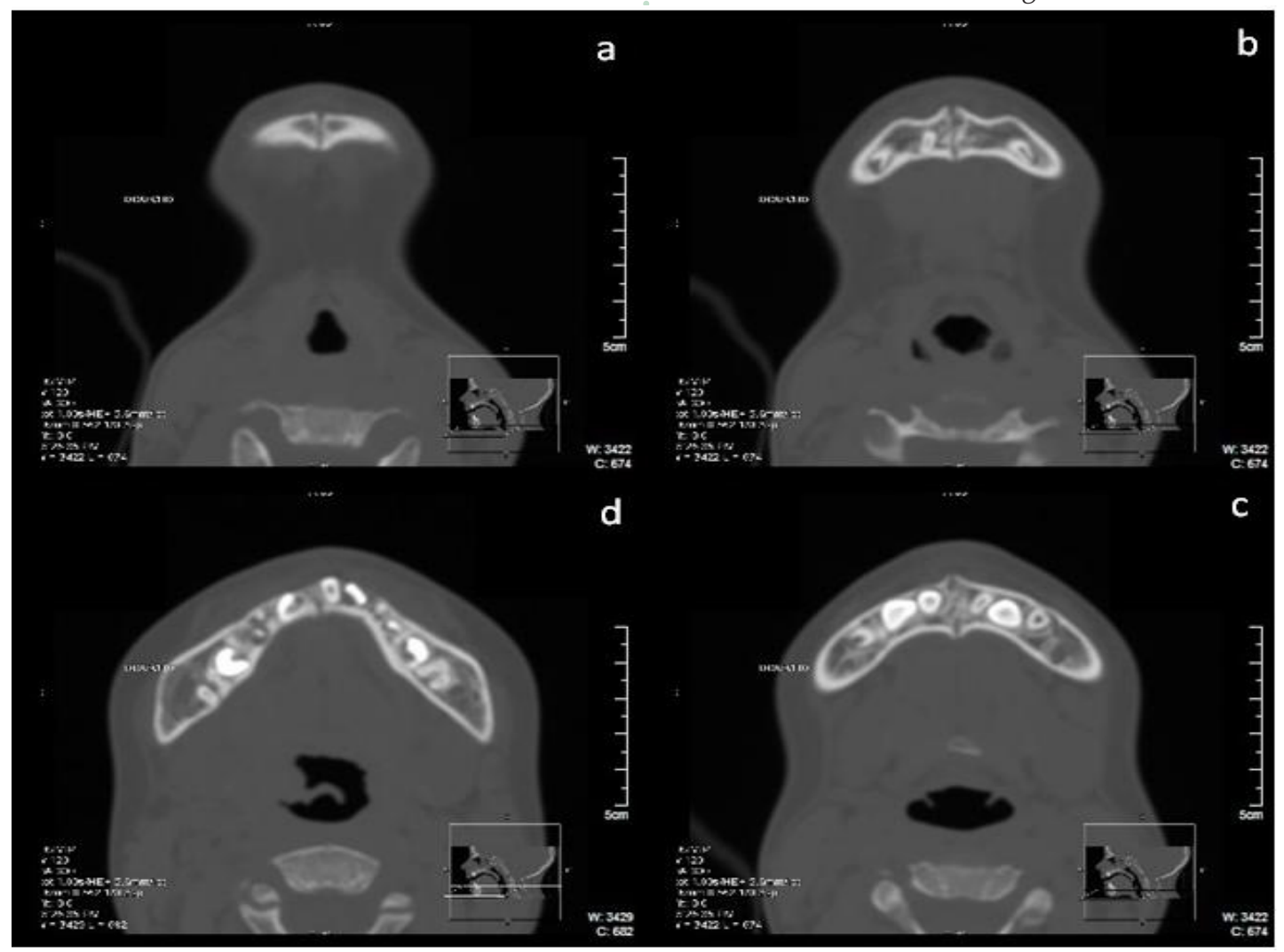


Figura 4. Reconstrucción de tomografía computarizada en 2D en el plano coronal, donde $4^{a}$ representa la vista más anterior y la 4d la más posterior. Se evidencia en $4 a$ solución de continuidad del borde inferior de la mandíbula e imagen hipodensa que se extiende desde dl mismo hacia un tercio del cuerpo mandibular en sentido ínfero-superior. En vista $4 b$ a $4 d$ la imagen hipodensa de bordes corticalizados se extiende desde el borde inferior de la mandíbula hacia el reborde alveolar.


ronal, se observa en la Figura 4a la solución de continuidad en el borde inferior de la mandíbula que se extiende discretamente en el cuerpo mandibular, mientras que en las Figuras 3b-3d se observa una imagen hipodensa que se extiende desde el borde inferior hasta la cresta alveolar con márgenes corticalizados. Las reconstrucciones volumétricas en 3D (Figura 5) ilustran los hallazgos descritos.

\section{DISCUSIÓN}

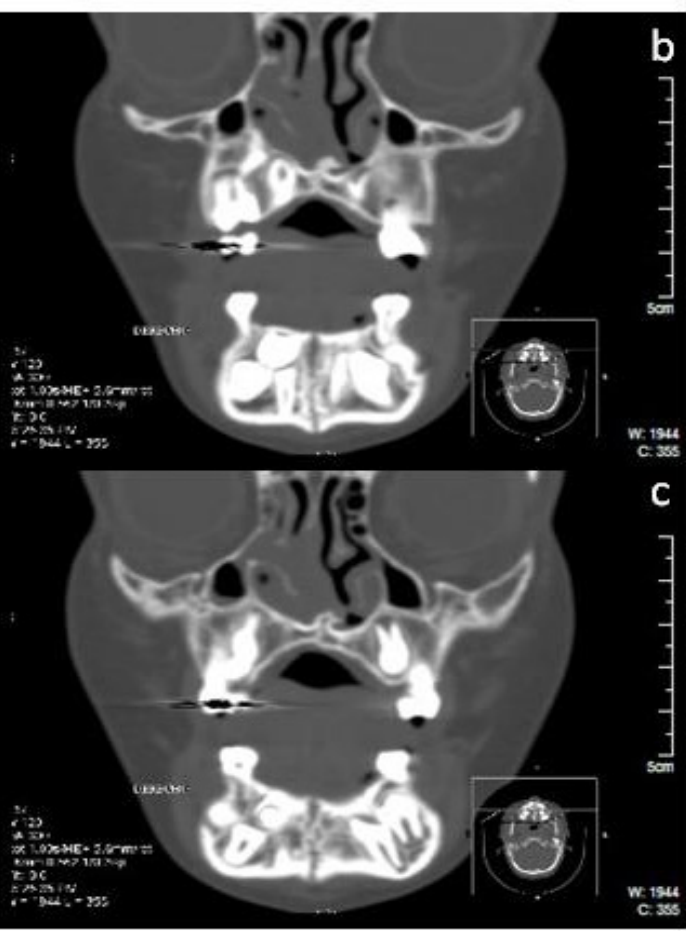

Las anomalías de desarrollo de las estructuras derivadas de la mitad superior del primer arco branquial son comunes, resultando en alteraciones como el labio o el paladar hendido. Sin embargo, el desarrollo anormal de las derivadas de la mitad inferior es inusual ${ }^{1,3,7}$. Embriológicamente, la mandíbula se desarrolla a partir del cartílago del primer arco faríngeo, el proceso mandibular, conocido como cartílago de Meckel, derivándose de la osificación de la membrana osteogénica formada por la

Figura 5. Reconstrucciones tomográficas en 3D. 3a Vista anterior y 3b. Vista posterior.

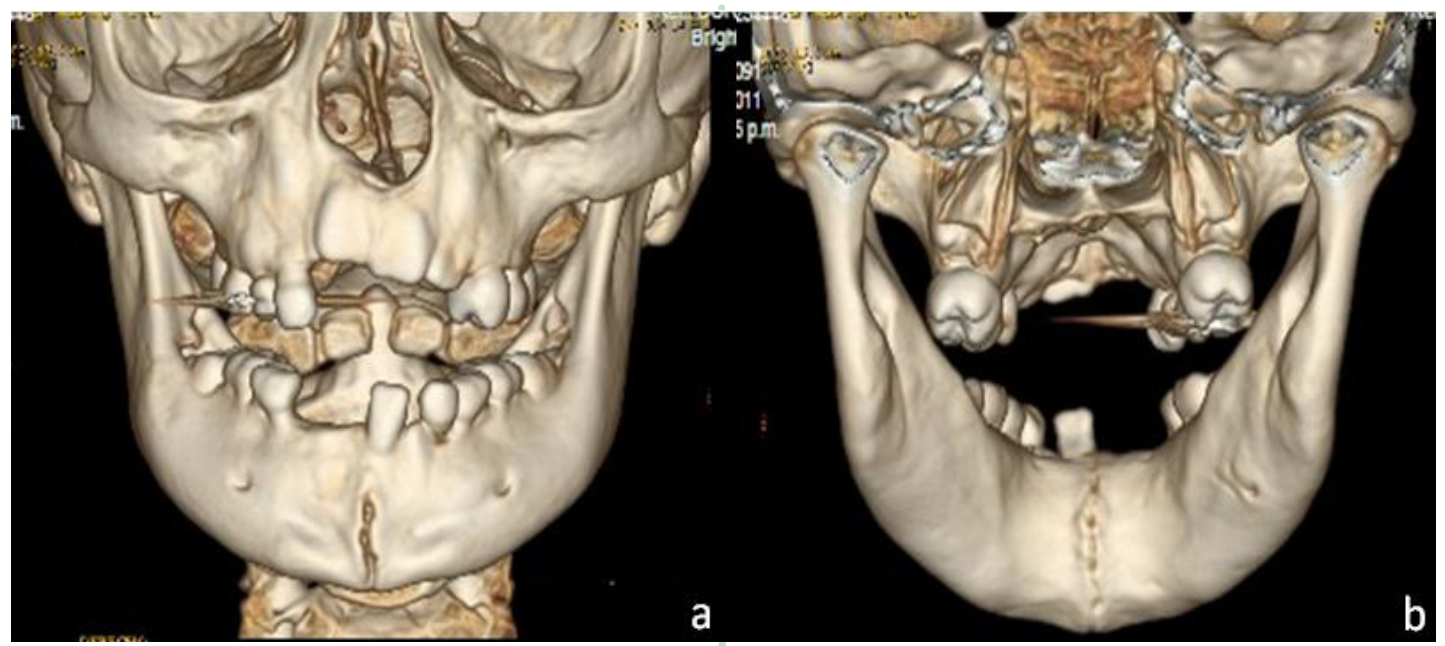

HERNÁNDEZ-ANDARA A ORTEGA-PERTUZ AI GONZÁLEZ C

HALLAZGO

IMAGENOLÓGICO DE HENDIDURA

MANDIBULAR MEDIA INCOMPLETA:

REPORTE DE UN CASO

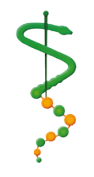

REV, ODONTOL.

UNIV. CID. SÃO

PAULO

2015; $27(2)$ :

156-62, MAIO-AGO 
HERNÁNDEZ-ANDARA A

ORTEGA-PERTUZ AI

GONZÁLEZ C

HALLAZGO

IMAGENOLÓGICO

DE HENDIDURA

MANDIBULAR MEDIA

INCOMPLETA:

REPORTE DE UN CASO

$160 \ldots$

REV, ODONTOL.

UNIV, CID, SÃO

PAULO

$2015 ; 27(2): 156-$

62, MAIO-AGO condensación ectomesenquimal que se produce a los 36-38 días de desarrollo. En la región del mentón, a cada lado de la sínfisis aparecen uno o dos pequeños cartílagos y hacia la séptima semana de desarrollo intrauterino se inicia la osificación endocondral para formar numerosos huececillos. Dichos huesecillos se incorporan al hueso intramembranoso cuando la sínfisis pasa de constituir una sindemostosis a una sinostosis durante el primer año de vida postnatal ${ }^{2,8}$.

Se han propuesto diversas hipótesis sobre la patogénesis de esta alteración. Algunos autores presumen que la falla en la fusión de la mandíbula se debe a que no se produce la penetración mesodérmica en las estructuras de la línea media de la porción mandibular del primer arco branquial ${ }^{2,8}$. Esta falla puede deberse a una deficiencia o crecimiento retardado del mesénquima, lo que parece ocurrir al término de la quinta o al inicio de la sexta semanas de gestación ${ }^{8}$.

Por su parte, Oostrom et al. ${ }^{9}$ señalaron que solo uno de los primeros arcos branquiales se desarrolla durante el periodo embriogénico temprano, y que los dos procesos mandibulares se desarrollan dentro de ésta estructura, dichos procesos mandibulares no se fusionan sino que emergen durante el periodo embriogénico tardío (después de la séptima semana de desarrollo). En el mismo periodo, se produce la formación del labio inferior y el proceso alveolar con alargamiento y surgimiento de la membrana osteogénica en cada proceso mandibular, resultando en la formación de la mandíbula y su sínfisis. Por ello, la hipoplasia del proceso mandibular durante los periodos embriológicos tempranos conduce a las hendiduras mandibulares más severas, las cuales se extienden al cuello, mientras que si se presentan en un periodo embrionario más tardío, la hendidura es menos severa. Estos autores también propusieron que las perturbaciones en los centros de formación ósea de la mandíbula resultan en una hendidura del tejido blando y óseo, en cuanto que defectos en el proceso emergente produce una muesca en el bermellóndel labio o una mayor hendidura del labio o una mayor hendidura del labio inferior con o sin comprometimiento del proceso alveolar de la mandíbula.

Existe un amplio espectro en la presentación de esta alteración, lo cual parece estar relacionado con el momento de la embriogénesis en el cual se produce ${ }^{9}$. En el labio se ha reportado desde la presencia de una muesca en el bermellón hasta la completa escisión ${ }^{10}$. La hendidura del labio suele estar acompañada de la hendidura de la mandíbula con movilidad de los segmentos resultantes y agenesias dentarias 7,11 . En la lengua se han descrito alteraciones que varían desde una punta bífida, anquiloglosia con adhesión a los márgenes de la hendidura mandibular a hipoplasia ${ }^{1-3}$. En las formas severas el hueso hioides, la epiglotis y el cartílago tiroideo pueden estar hendidos o completamente ausentes. Con relación al cuello, los haces musculares pueden estar atróficos y estar representados por tejido fibroso que causa la contractura del mismo. Se ha descrito la separación de las clavículas, mientras que se ha reportado un manubrio del esternón bífido o ausente ${ }^{12}$.

Otras anomalías faciales que se han asociado a esta condición son el paladar hendido, anomalía Pier Robin, microsomia hemifacial, quistes dermoides en la nariz o el mentón, alteraciones de la nariz y del pabellón de la oreja. Estudios han resaltado la presencia de anomalías congénitas del corazón como el ventrículo simple y la transposición de los grandes vasos, entre otros, así como anomalías en las manos (sindactilia, braquidactilia) y de los pies (pie equinovaro, ectrodactilia) ${ }^{4,}$ 13-15.

En el presente caso no se observó una muesca o hendidura labial, pero si se constató la presencia de una hendidura mandibular media incompleta, sin movilidad de los segmentos. Los elementos dentarios próximos a la misma, se encontraron presentes y retenidos. Se consideró durante el análisis de las imágenes, la posibilidad de que la misma fuera compatible con un trazo de fractura, sin embargo, la ausencia de heridas cutáneas y la corticalización de los márgenes observada tanto en la radiografía panorámica como en las imágenes de TC, sin que se evidenciara la aposición de tejido óseo, posibilitó 
el descarte de la misma. De forma similar, Freitas et $\mathrm{al}^{2}$, reportaron un caso de hendidura mandibular media, sin alteración del labio, lo que puede apoyar la hipótesis de la presencia de hendidura en el paciente, de manera que la alteración se produjo en él en un periodo tardío de la embriogénesis. Por otro lado, la evaluación mediante la TC posibilitó determinar la extensión de la hendidura en los tres planos, pudiendo identificar que no era completa en dirección ínfero-superior o vestíbulo-lingual, lo que no es posible determinar en una radiografía convencional.

\section{CONCLUSIÓN}

La hendidura mandibular media puede presentarse sin la alteración del labio inferior siendo que en el paciente estudiado, la misma fue identificada como un hallazgo casual en la radiografía panorámica. La TC permitió la evaluación de la extensión de la hendidura en los tres planos espaciales, posibilitando su caracterización.

\section{REFERÊNCIAS}

1. Almeida LE, Ulbrich L, Togni F. Mandible cleft: report of a case and review of the literature. J Oral Maxillofac Surg 2002 Jun;60(6):681-4.

2. Freitas $\mathrm{R}$, Oliveira $\mathrm{G}$, Alonso $\mathrm{N}$, Forte A, Busato L, Persing J. Tessier number 30 clefts: surgical corrections of a rare malformation. Rev Bras Cir Craniomaxilofac 2010 13(1):12-7.

3. Guttikonda LK, Nadella KR, Uppaluru V, Kodali RM, Nallamothu R. Nonsyndromic mandibular symphysis cleft. Case Rep Dent $20142014(682163$.

4. Tafreshi M, Aminolsharieh Najafi S, Hasheminejad R, Mirfazeli A, Shafiee A. Tessier number 30 clefts with congenital heart defects. Iran Red Crescent Med J 2015 Mar; 17(3):e19078.

5. Rajion Z. Craniofacial computed tomography imaging: a review. Arch Orofac Scie 2006 1(5-8.

6. Picolli P, Menezes L, Brucker M, Azeredo F, Rizzanato S. Assessment of alveolar defect volume in unilateral cleft lip and palate patients using a free software program. Open / Stomatol 2013 3(9A):31-6.
7. Prakash A, Parelkar SV, Oak SN, Gupta RK, Sanghvi BV. Giant epignathus with midline mandibular cleft: Insights in embryology and management. Ann Maxillofac Surg 2012 Jan;2(1):56-9.

8. Fujino $\mathrm{H}$, Kyoshoin $\mathrm{Y}$, Katsuki T. Median cleft of the lower lip, mandible, and tongue with midline cervical cord: a case report. Cleft Palate J 1970 Apr;7(679-84.

9. Oostrom CA, Vermeij-Keers C, Gilbert PM, van der Meulen JC. Median cleft of the lower lip and mandible: case reports, a new embryologic hypothesis, and subdivision. Plast Reconstr Surg 1996 Feb;97(2):313-20.

10. Rana R, Puri V, Thakkur R, Baliarsing A. Median cleft of mandible and lower lip with ankyloglossia and ectopic minor salivary gland on tongue. Indian J Plast Surg 2004 37(1):67-70.

11. Bhattacharyya NC, Kalita K, Gogoi M, Deuri PK. Tessier 30 facial cleft. J Indian Assoc Pediatr Surg 2012 Apr;17(2):75-7.
HERNÁNDEZ-ANDARA A ORTEGA-PERTUZ AI GONZÁLEZ C

HALLAZGO IMAGENOLÓGICO DE HENDIDURA MANDIBULAR MEDIA INCOMPLETA: REPORTE DE UN CASO

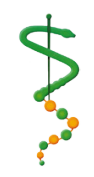

REV, ODONTOL.

UNIV. CID, SÃo

PAULO

2015; $27(2)$ :

156-62, MAIO-AGO 
HERNÁNDEZ-ANDARA A ORTEGA-PERTUZ AI GONZÁLEZ C

HALLAZGO

IMAGENOLÓGICO

DE HENDIDURA

MANDIBULAR MEDIA

INCOMPLETA:

REPORTE DE UN CASO
12. Bordoloi U. Tessier No 30 presenting with median cleft of the lower lip and mandible, ankyloglosia and midline cervical web causing neck contracture. J Evo Med Dent Sci 2012 Oct.;1(4):606-9.

13. Ishii M, Ishii $Y$, Moriyama T, Enomoto S, Ono T, Ohyama K, et al. Seventeen-year follow-up of a patient with median cleft of the lower lip, mandible, and tongue with flexion contracture: a case report. Cleft Palate Craniofac J 2002 Sep;39(5):555-9.
14. Eppley BL, van Aalst JA, Robey A, Havlik RJ, Sadove AM. The spectrum of orofacial clefting. Plast Reconstr Surg 2005 Jun;115(7):101e-14e.

15. Desai A, Kumar N, Pavate S, Ramarathian D. Incomplete median cleft of lower lip with ankyloglossia. J Cleft Lip Palate Craniofac Anom 2015 Feb;2(1):63-5.

Recebido em 16/11/2015

Aceito em 16/11/2015
REV, ODONTOL. UNIV, CID, SÃO PAULO $2015 ; 27(2): 156-$ 62. MAIO-AGO 